\title{
Modeling of hybrid method as combined spark plasma sintering and hot pressing physical processes
}

\author{
Andrey V. Smirnovi,a, Denis I. Yushin ${ }^{1}$, Igor A. Zverev ${ }^{1}$, Andrey R. Maslov ${ }^{1}$ \\ AND RAMON TORRECILLAS ${ }^{1,2}$ \\ 1 Moscow State University of Technology "STANKIN", 1 Vadkovsky per., 127994 Moscow GSP-4, Russian Federation \\ 2 Nanomaterials and Nanotechnology Research Center (CINN), Oviedo, Spain
}

Received 24 September 2015, Accepted 15 October 2015

\begin{abstract}
The numerical models of sintering processes from WC powders by the methods of spark plasma sintering and hybrid method as a combination of spark plasma sintering and hot pressing are developed. The models are tested with experimental data of the processes.
\end{abstract}

Key words: Spark-plasma sintering / modeling of physical processes / finite element method / hot pressing / nanostructured powder materials

\section{Introduction}

At present, creation and implementation of nanostructured metal and ceramic materials is one of the most interesting subject from a practical and scientific standpoint. It can be explained by the fact that such materials possess unique physical and mechanical, as well as physical and chemical properties. For this reason the improvement of the existing methods for processing such materials and development of new ones is a matter of current interest.

The most effective and popular technology that allows to make products of such materials is spark-plasma sintering (SPS). SPS technology is a developing variety of hot-pressing method of powder materials, in which pulsing direct current is used for high-speed heating of matrix and powder. SPS saves time for the synthesis of samples and increases the quality of the sintered material. Particularly, this method allows preserving nano- and microstructure in nano-dispersed powder materials as a result of their consolidation.

Heating during the SPS occurs at the time of direct pulsing current flow through matrix elements and treated material (Joule heating) or hybrid heating (HH). In case of hybrid heating, the SPS technology is combined with hot-pressing (HP) technology, allowing heating due to induction element. Such combination is applied for the compensation of temperature gradient during sintering made by SPS method, directed inside-out. The additional induction element creates temperature gradient with oppo-

\footnotetext{
a Corresponding author: lecast@stankin.ru
}

site direction allowing uniform temperatures distribution along the section of the product.

However, a large-scale implementation of technological methods, such as SPS and SPS + HP, is limited today due to low level of theoretical description of the process. Nowadays, the state of theoretical knowledge on physical processes occurring inside powder compact during sparkplasma sintering is not adequate for the effective development and improvement of SPS process.

This paper provides the results of modeling of temperature distribution and mechanical stress in the product and matrix for the SPS process carried out at the unit (FCT Systeme GmbH, model KCE-FCT H-HP D 25) using $\mathrm{HH}$ or without it.

\section{Experimentation and modeling}

A simplified geometrical model of technological processes was developed for the adjustment of numerical modeling and analysis by the finite-element method (FEM) of SPS process and model testing. The necessary properties of the materials mentioned in the paper were borrowed from literature sources [1-5].

When developing the technological process of any sample consolidation using SPS method (or its hybrid variant) at the unit (FCT, H-HP D 25), the following input parameters are set (process control parameters): sample sintering temperature $\left(\mathrm{C}^{\circ}\right)$, press-form temperature $\left(\mathrm{C}^{\circ}\right)$, pressing power $(\mathrm{kN})$; sample holding time at 


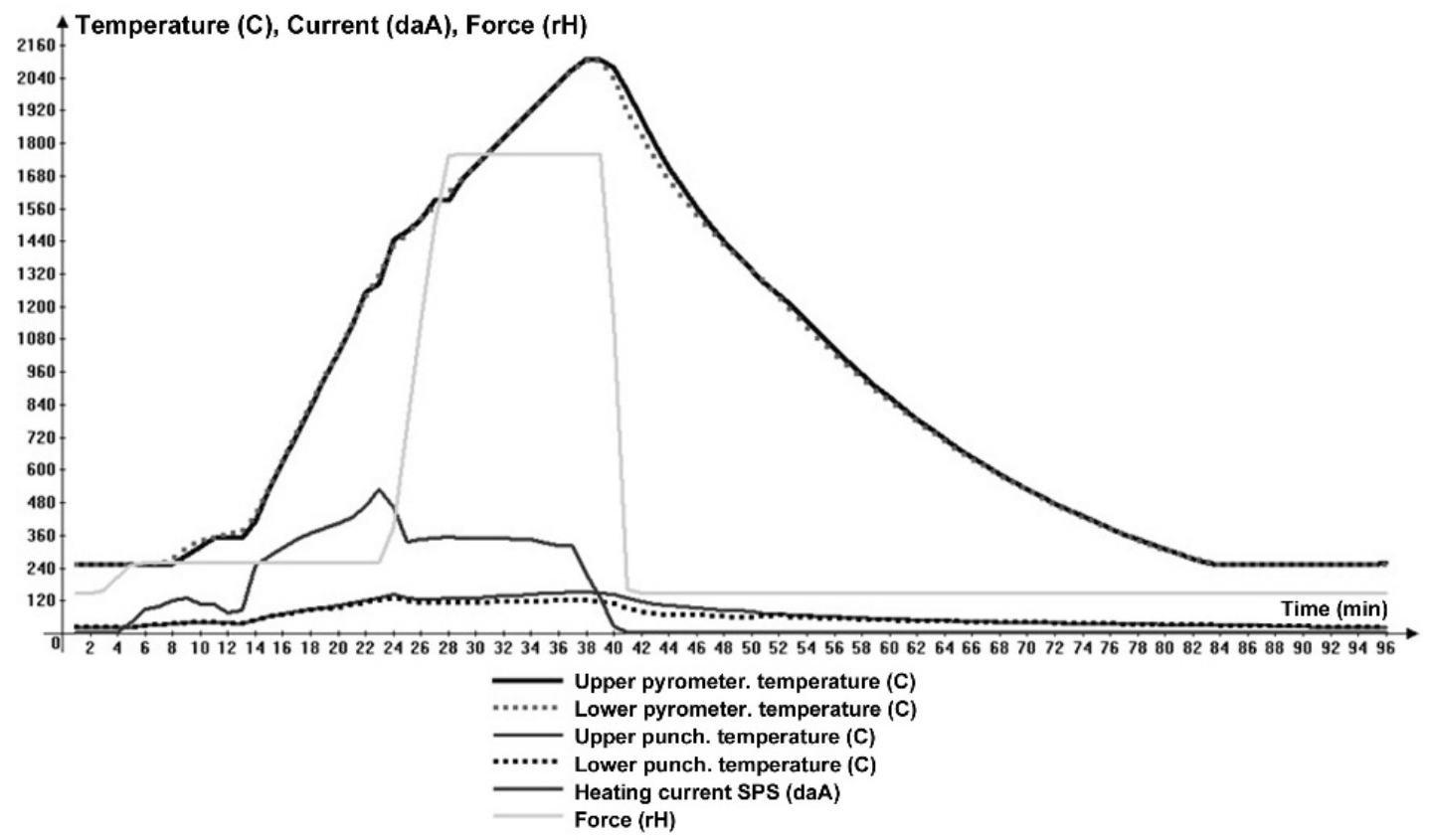

Fig. 1. Parameters of sintering process of a sample with $80 \mathrm{~mm}$ diameter made of tungsten carbide powder (WC) by SPS + HP method.

sintering temperature (s); time of defined force application $(\mathrm{s})$. For numerical sintering process modelling, it is necessary to set other input data, such as source sample temperature, equipment and chamber temperature $\left(\mathrm{C}^{\circ}\right)$; direct current of SPS heating (A); electric voltage (potential) of SPS heating $(\mathrm{V})$; pressing power $(\mathrm{kN})$ or pressure $(\mathrm{Pa})$; time of current, voltage, pressing force or compacting pressure application ( $\mathrm{s})$.

The necessary data for modelling by finite element method are recorded in the unit computer memory during sample sintering. The data reflecting the time of real sintering process are subsequently used to compare the results obtained during the numerical FEM modeling with experimental data and to correct the numerical model of the process under study. The data recorded during the process of consolidation of the sample with the diameter of $80 \mathrm{~mm}$ made of tungsten carbide powder by SPS + HP method are represented graphically in Figure 1.

The theoretical model as for consolidation processes of powder materials by SPS method is based on the ordinary differential equations in partial derivatives. It describes non-stationary heating and heat-transmission processes in the adjoint thermoelectrical problems, strainstress states of the system caused by the impact of thermal expansion of the system's materials, as well as the application of external compacting pressure and powder compact compression processes under the influence of temperature and pressure.

The input control parameters for this model are boundary conditions and loads. The equipment and sample material properties outlined as a separate file code of the library of materials in $A N S Y S$ are also very significant input data that greatly affect the modeling results.
The boundary conditions in this model are as follows: input temperature $(\mathrm{K})$; volume of vacuum chamber $\left(\mathrm{m}^{3}\right)$; emission of system elements; Stefan-Boltzmann constant $\left(\mathrm{W} \cdot \mathrm{m}^{-2} \cdot \mathrm{K}^{-4}\right)$.

The loads in this model (when conducting thermoelectrical analysis) are the following: direct current (A); voltage $(\mathrm{V})$.

The model under consideration is non-stationary and, as an additional input parameter before numerical modeling, the time (s) of load action (current or voltage) should be set.

In addition to the numerical analysis of mechanical and temperature stresses in the equipment and the sintered sample, the given numerical model allows to perform complex analysis of stress-strain state of the whole system due to the possibility to calculate the distribution of temperatures and mechanical deformations.

The numerical model for SPS modeling of powdered materials' sintering by hybrid method SPS + HP has been created in the COMSOL Multiphysics program environment. This program allows to model complex by multiphysical adjoint models in interactive interface. For numerical modeling of hybrid sintering included in the above-mentioned model it is necessary to solve the following interconnected tasks: Joule heating of the equipment (press tools and plugs) and the sample during heavy direct current flow (heating in SPS mode); inductive heating of electrically conductive system elements by cylindrical induction heater working on alternating current (heating in hybrid mode); stress-strain state of the equipment and the sample determined by mechanical and temperature stresses caused by heating and application of compacting pressure; powder contraction under the influence of temperature and pressure. 
A.V. Smirnov et al.: Mechanics \& Industry 16, 712 (2015)

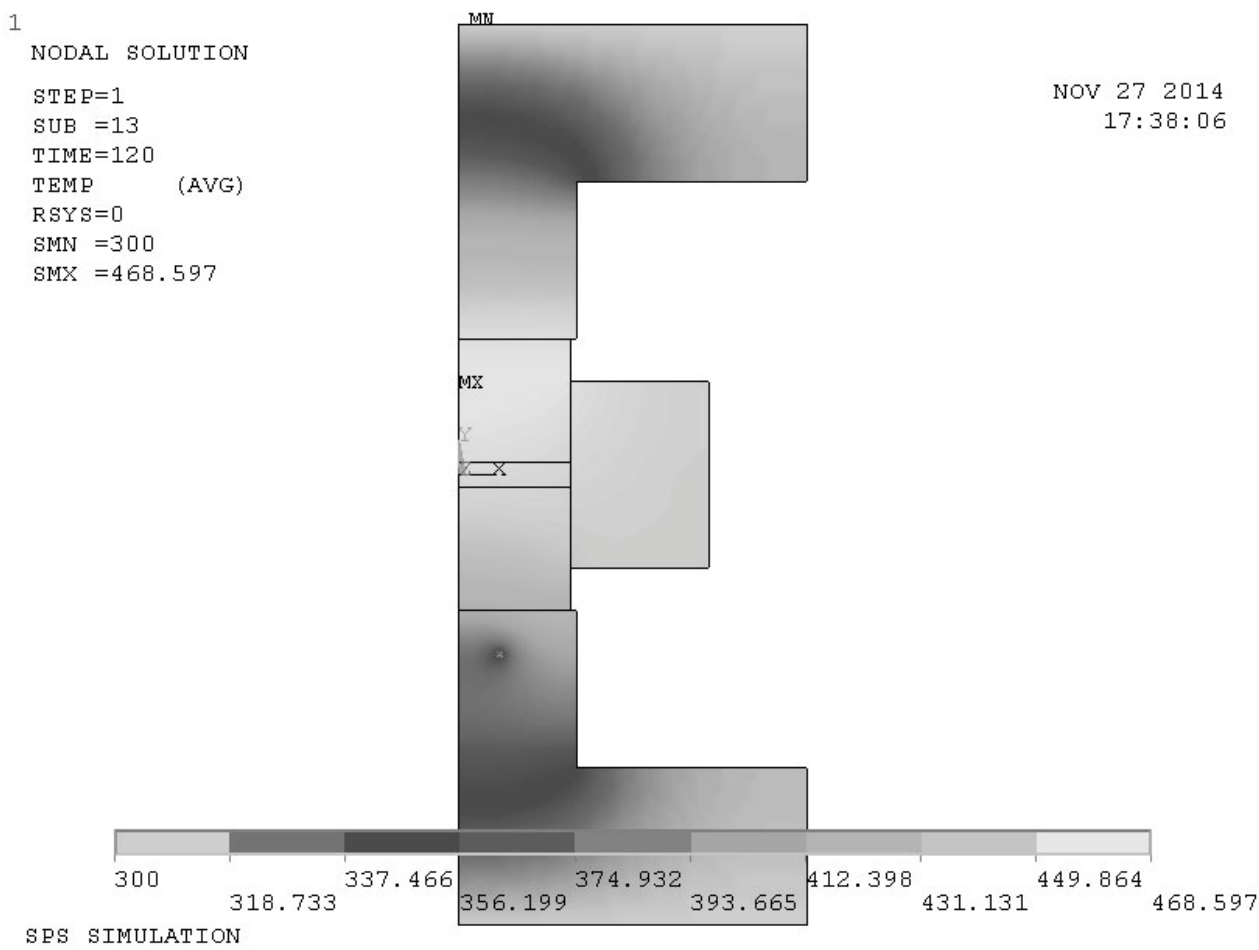

Fig. 2. Temperatures distribution after current application $1200 \mathrm{~A}$ during $120 \mathrm{~s}$ at the initial temperature of $523 \mathrm{~K}$.

Figure 2 shows temperatures distribution in the model after current application 1200 A during 120 s. The highest temperature $(750 \mathrm{~K})$ is reached in the punches, but not in the sample or pressing tool. It can be explained by high efficiency of tungsten carbide powder that is why current flows directly through the sample and heats punches and the sample intensively starting from the center. At the beginning of tungsten carbide sintering process there exists a significant temperature gradient in the sample. The data on modeling correlate to the experimental data recorded in the memory of the SPS unit, to a precision of $\pm 15-20 \%$, which can be explained by the following: first, for comparison with the experiment the data recorded during hybrid sintering (not data recorded during SPS method) were used. Second, the data on the properties of equipment and sintering sample materials used during the modeling were borrowed from literature sources, but not from real data as to the properties of certain materials used during sintering.

Figure 3 shows temperatures distribution in the model after current application of $5000 \mathrm{~A}$ in $120 \mathrm{~s}$. It corresponds to sintering process transition to the final heating stage of powder compact. As shown in the figure above, temperatures distribution along the sample section is homogenous. The error of modeling results compared to experimental data is less than $10 \%$.

The results of numerical analysis of thermo-electrical task of Joule heating of the pressing tool, the punches, and powder compact used during the sintering of the sample made of tungsten carbide powder by SPS method were used for solving the adjoint problem of the distribution of temperatures and mechanical stresses in model under study (Fig. 4).

$3 \mathrm{D}$ solid model of hybrid sintering system is shown in Figure 5. This model includes graphite element intended for sintering samples with the diameter of $80 \mathrm{~mm}$ by SPS method, complemented with copper cylindrical induction heater, which is separated from graphite cover by thermal and electrical isolation graphite felt. The solid model is imported from the COMSOL Multiphysics software environment and is a geometrical model, which is to be further complemented by final mesh, boundary conditions and applied loads. The boundary conditions include: initial temperature of the model, emission capacity of system elements (heat losses caused by the emission in vacuum chamber), fixing elements of graphite cover, and induction heating element.

Applied loads in this model are as follows: direct current applied to graphite electrodes; alternative current applied to induction element; compacting pressure applied to graphite punch.

The properties of cover materials and the sample in the model were set similarly to those used in the SPS process model. Induction heating element material is made of copper and thermal insulated graphite foil; their properties were taken from the standard material library COMSOL Multiphysics.

\section{Results}

The results of model testing for numerical analysis of hybrid sintering have shown significant differences 
A.V. Smirnov et al.: Mechanics \& Industry 16, 712 (2015)

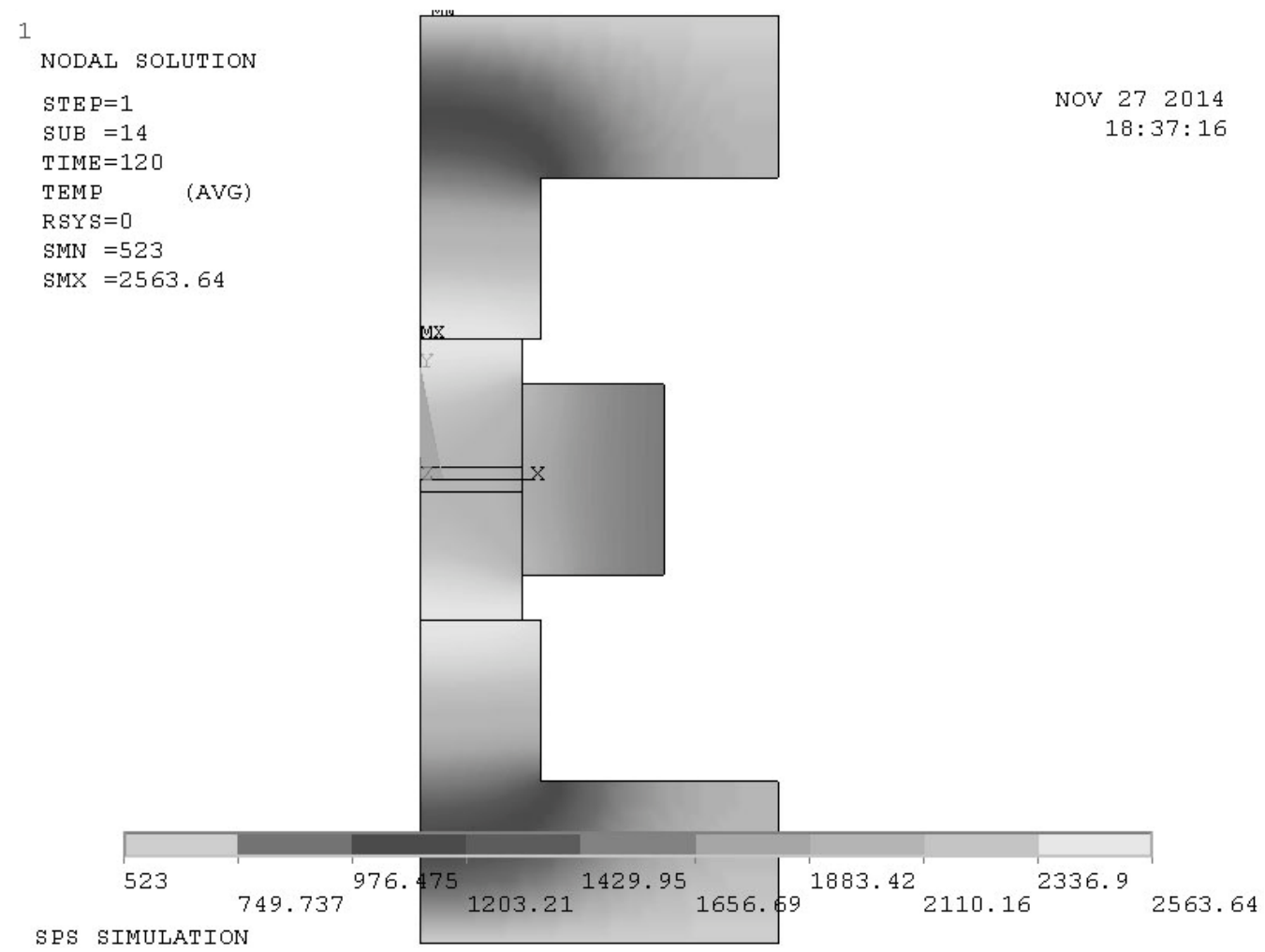

Fig. 3. Temperatures distribution after current application $5000 \mathrm{~A}$ in $120 \mathrm{~s}$ at the initial temperature of $523 \mathrm{~K}$.

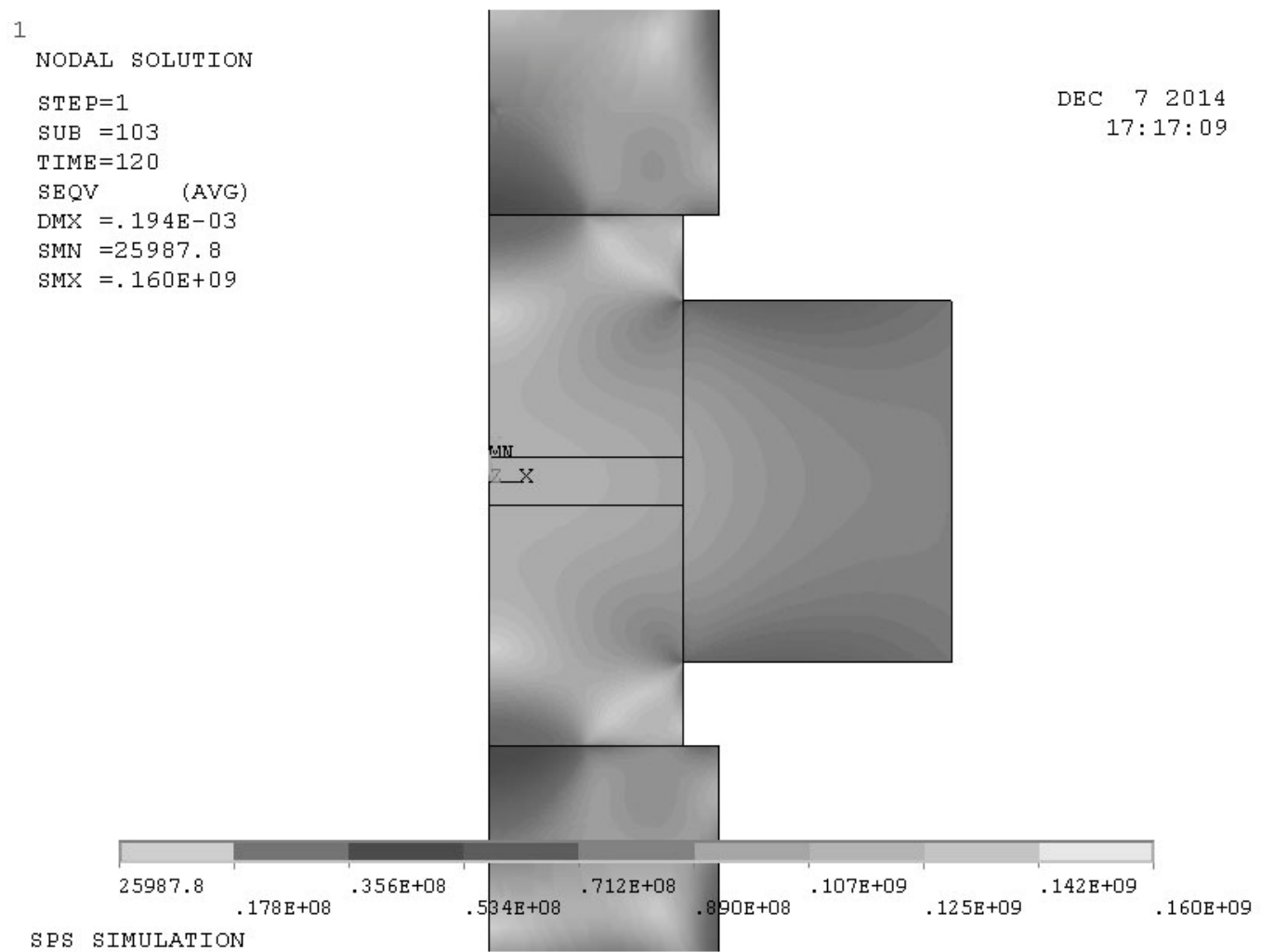

Fig. 4. Distribution of mechanical stresses at compacting pressure $P=394.7 \mathrm{kPa}$, current $I=1200 \mathrm{~A}$ in $120 \mathrm{~s}$. 


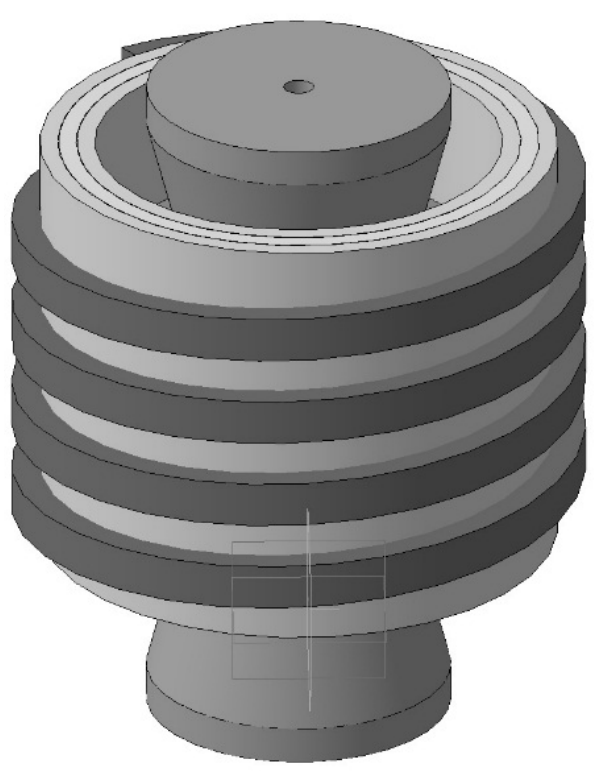

Fig. 5. Cover and induction heating element model for SPS and HP in assembly.

in temperatures distribution in the volume of samples, punches, and press tools. Hybrid heating of SPS and HP (Fig. 6a) provides a more even distribution of temperatures in press tool and the sintered powder compact as compared to the temperature gradient, which is present during SPS sintering (Fig. 6b) under similar conditions.

These results are in accord with the data received during sintering of the sample with $80 \mathrm{~mm}$ diameter. The sample is made of tungsten carbide at the SPS unit (see Fig. 1), which shows almost identical temperature values given by the upper pyrometer (measuring temperature in close proximity to powder compact) and the side pyrometer measuring temperature on the surface of the press tool.

Many scientific papers from all over the world are dedicated to the research of consolidation of complex-shape powder samples by SPS method. Based on the analysis of the main studies $[6,7]$, we can define two conceptual directions concerning the development of complex samples using the SPS method.

The first direction is consolidation of powder compact sample placed into press tool of appropriate configuration. Using such approach depending on the complexity of the shape of sintered sample the technological covers with two or more complex-shaped samples are used.

The second direction is obtaining the right sample shape by combining SPS with extrusion or pressing. The initial material includes a sintered sample having simple shape [8].

However for implementation of both types of this technological process one needs special technological cover and equipment as punches and press tools of given parameters.

Designing such special instruments for sintering is a very complicated process so far understudied. The main requirements for the punches and press tools for sintering

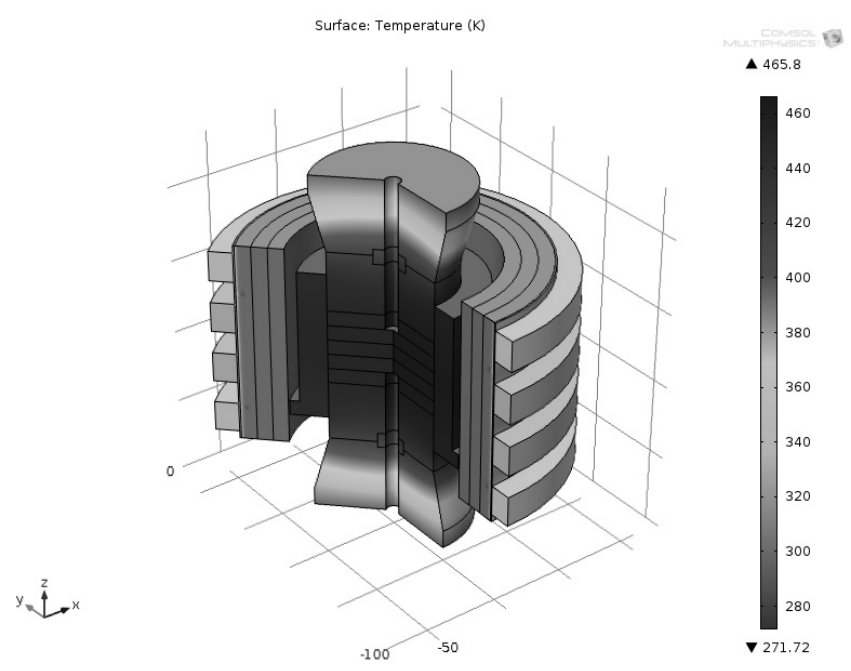

(a)

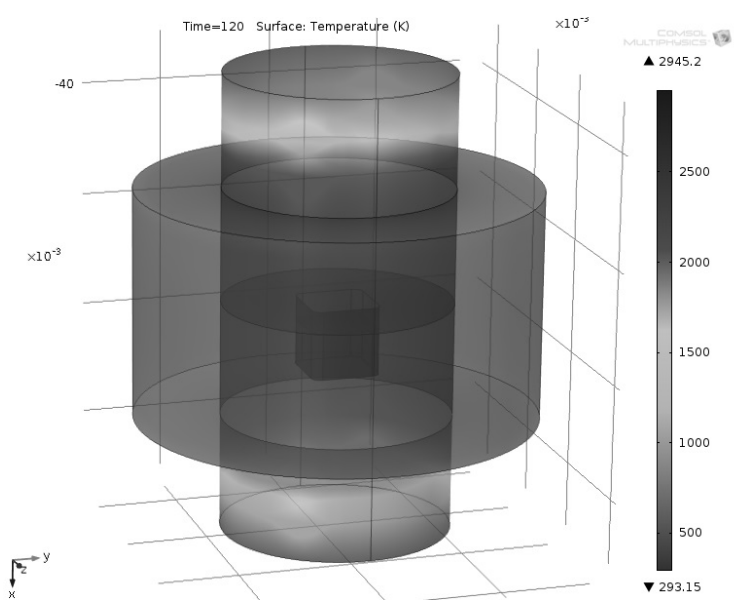

(b)

Fig. 6. Temperature distribution in graphite cover and sample during the process of: (a) SPS + HP, (b) SPS. Current $I=$ $1500 \mathrm{~A}, t=100 \mathrm{~s}$.

of the samples of various shapes are the provision of highquality consolidation process (equal to the gradient density or sample grain dimensions) during the formation of the sample from powder compact; and the respective capacity needed for presses (for both variants). That is why the design of punches includes numerical FEM modeling for calculations of the systems' strength and calculation of temperature and electrical fields during consolidation.

When the work above is performed, numerical models intended for FEM analysis of temperatures distribution, current, heat flows density, strain-stress state in the press tool and in the sample were carried out either for direct consolidation of the complex-shaped sample made of powder compact, or for the combination of SPS and pressing. Each model has been developed and tested within the software environment for numerical modeling COMSOL Multiphysics.

Direct consolidation powder compact model (Fig. 7) was developed for sintering of the samples having 


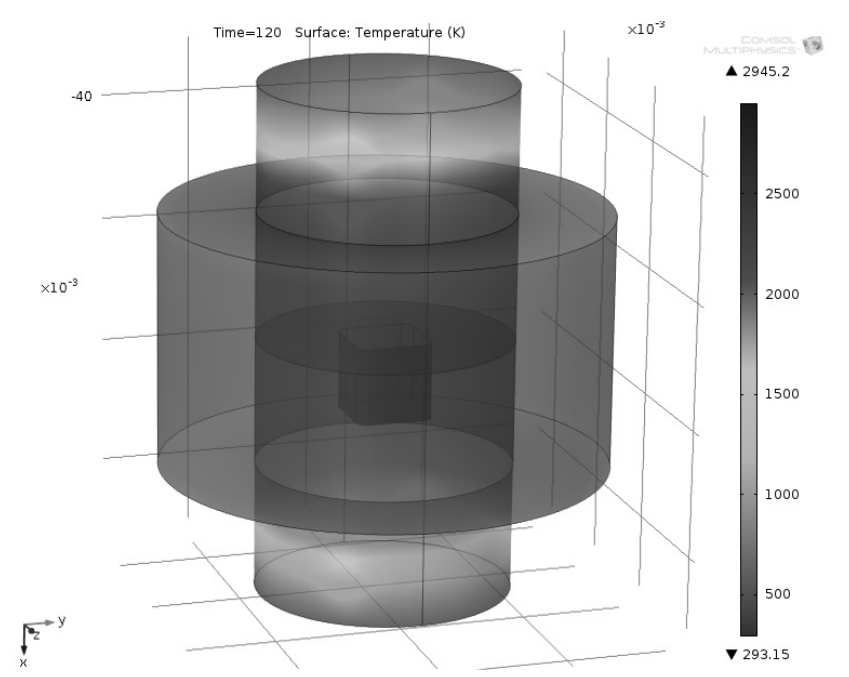

Fig. 7. Numerical model of direct consolidation of square plate from $W C$ by SPS method.

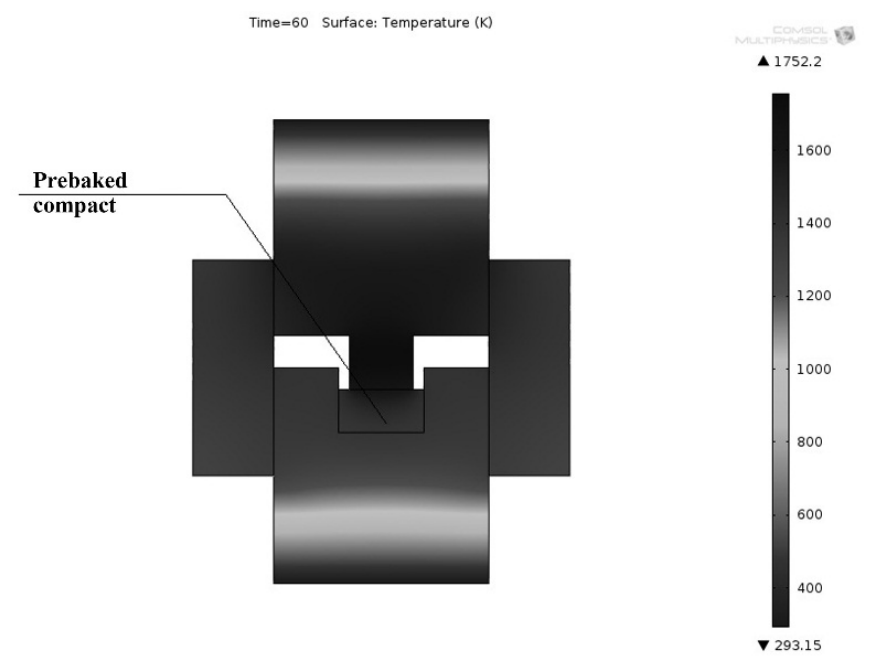

Fig. 8. Numerical model of plug production from TiAlcomposition by SPS method combined with extrusion.

rectangle and rhomboid shape with spherical radius at vertex (geometrically, these samples are identical to standard detachable cutting plates for lathe work) as well as the sample having plug shape.

The main materials of the press tool and plugs in these models include the same graphite used during the consolidation of tungsten carbide sample; and for the sample modeling was pure tungsten carbide as well as pure aluminum oxide $\mathrm{Al}_{2} \mathrm{O}_{3}$.

A numerical model of complex-shaped sample production process from previously sintered material (Fig. 8) was developed for shaping by hybrid SPS method and by the extrusion of the samples having sleeve shape and made of titanium and aluminum.

Calculation of strength of load-up condition during spark-plasma sintering of complex-shaped samples is necessary for defining of the shape and size of the punches

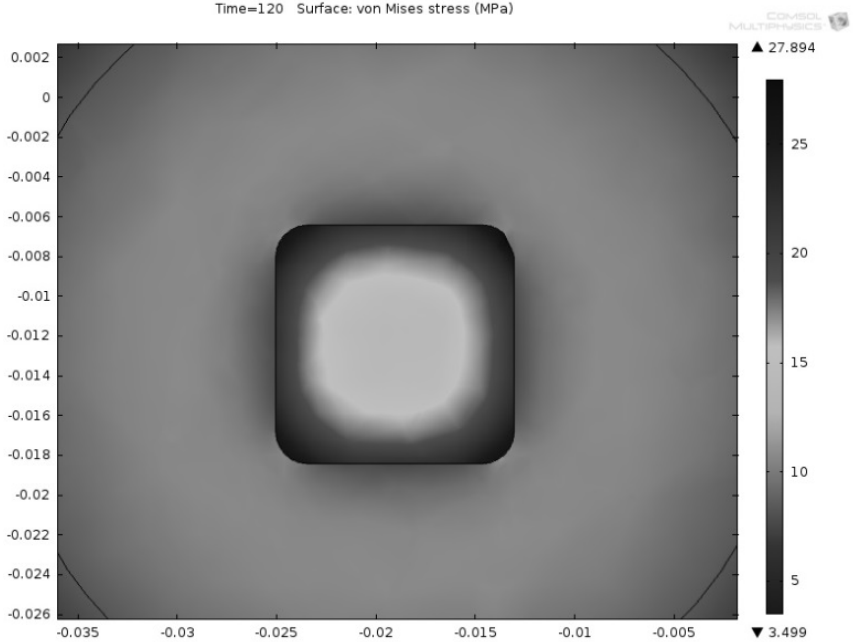

(a)

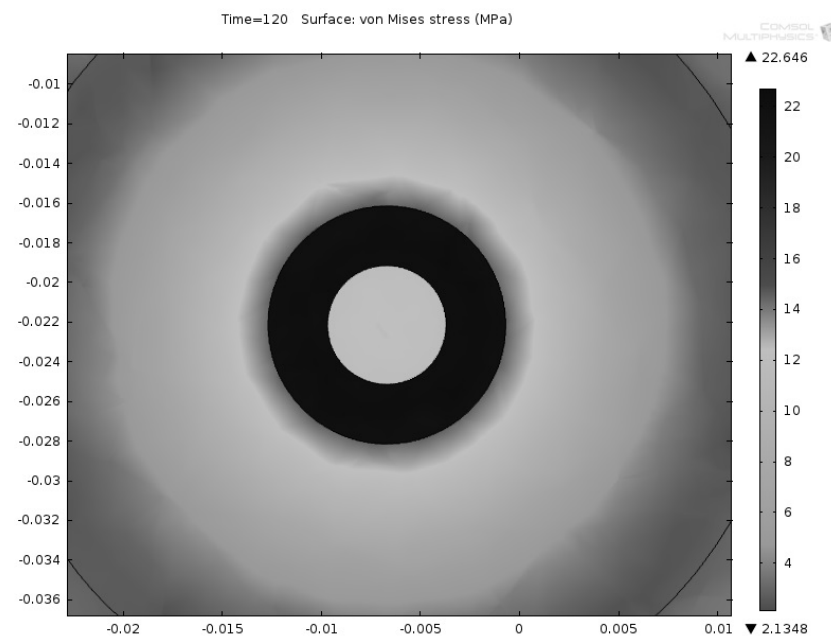

(b)

Fig. 9. Distribution of equivalent voltages during consolidation and shaping: (a) plate of $\mathrm{WC}$, (b) plug of $\mathrm{Al}_{2} \mathrm{O}_{3}$.

and press tool and for selection and projection of the material for the elements of technological system. The most popular method for strength calculation includes the following sequence of actions: creating of numerical model of sintering process, and shaping (or simultaneous sintering and shaping); modeling of FEM and identification of equivalent voltages distribution in the volumes of samples, punches, and press tools; analysis of stressed condition for the search of stress sources and identification of the largest values of voltage in the system; carrying out experimental testing and comparison of results of numerical modeling and the data received during the experiment.

The following conclusion based on the analysis of the numerical modeling of load-up condition during sintering the samples made of tungsten carbide powder and aluminum oxide in the shape of square and rhomboid section and ring-shaped plug: at these modes of loading-up in complex-shaped punches, there are no voltage values, which exceed $30 \mathrm{MPa}$ (see Figs. 9a and 9b). 

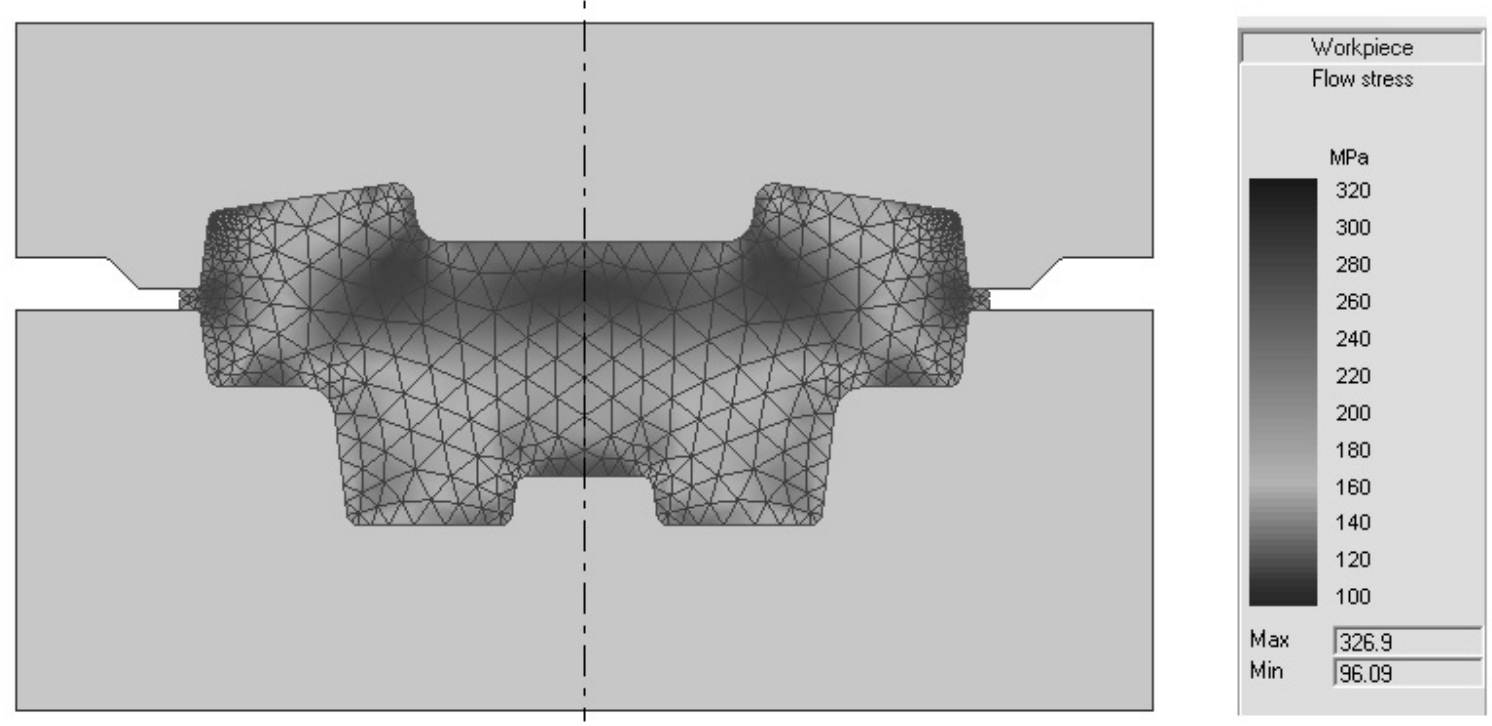

Fig. 10. Numerical modeling of voltage distribution during sintering by SPS method in previously sintered material TiAl.

The emerging voltage values are much lower than the limit strength of graphite used for the production of punches and press tools used in the laboratory. At this stage experiments are being prepared including sintering with shaping of complex-shaped samples from powder compact and the adjustment of parameters of the developed numerical models for their further correlation with the data received during the analysis of real SPS processes.

Strain-stress state occurring during the extrusion of previously sintered material heated by heavy current flow can be characterized by higher values of voltage and deformations than those during the consolidation and shaping processes (Fig. 10).

For the analysis of distributed temperature in the samples of the given geometrical shape and the respective equipment and cover during SPS sintering (either in case of SPS combined with shaping consolidation or in case of extrusion of previously sintered material), numerical models were created in $\mathrm{FE}$ analysis software COMSOL Multiphysics. The system heating was carried out by applying direct current $I=10000 \mathrm{~A}$ in $120 \mathrm{~s}$ to graphite plugs-electrodes. Temperatures distribution during sample sintering in the shape of square plate is shown in Figure 11. Square plate parameters are as follows: $12 \times 12 \mathrm{~mm}$ with radius at vertex $1.6 \mathrm{~mm}$, material is tungsten carbide (Fig. 11a) and aluminum oxide (Fig. 11b).

During the sintering of the plate made of electricityconductive tungsten carbide, maximum temperature of $2948 \mathrm{~K}$ generates directly in the sample and decreases along the gradient from the sample center to the graphite punch (Fig. 11a). At the vertex of square, minimal temperature of the sample can be observed, which is equal to $2800 \mathrm{~K}$. In the sample made of aluminum oxide, inverted gradient and lower maximal temperature can be observed (Fig. 11b). To decrease temperature difference, it is possi- ble to use hybrid heating SPS + HP or longer application of Joule heating.

\section{Conclusions}

In the course of the work described in the paper the modeling of physical processes by finite element method and those processes were achieved by spark-plasma sintering of nanostructured powder materials were investigated.

The numerical model for sintering of the sample made of tungsten carbide powder by SPS method within the ANSYS software environment with $A P D L$ programming language and the numerical model for sintering of the sample made of tungsten carbide powder by hybrid method SPS + HP within the COMSOL Multiphysics software environment were created. As well the numerical model for sintering processes by SPS method with shaping the samples having preset parameters was developed. The possibility of sintering of the samples with preset parameters was theoretically justified and explained.

The developed and tested numerical models will be used in future when the following issues are solved numerical analysis of FEM of mechanical and temperature stresses in the sample made of tungsten carbide and other materials; in development of equipment and cover at various stages of consolidation process by SPS and SPS + HP methods; numerical analysis of FEM of the distribution as to the density in the volume of samples made of tungsten carbide and other materials at various stages of consolidation process by SPS and SPS + HP methods; designing, testing and optimization of special technological equipment and cover (punches and press tools) for consolidation of the samples with the complex shape by SPS and SPS + HP methods. During the development of technological schemes for the design and production 
A.V. Smirnov et al.: Mechanics \& Industry 16, 712 (2015)

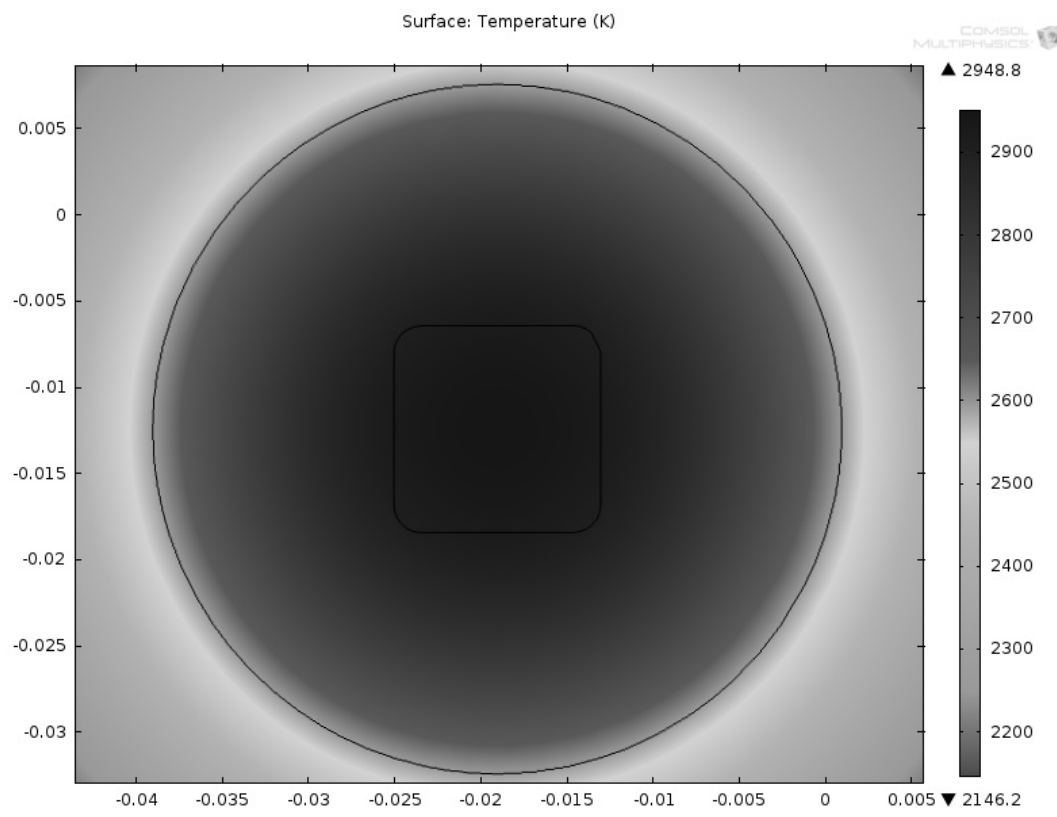

(a)

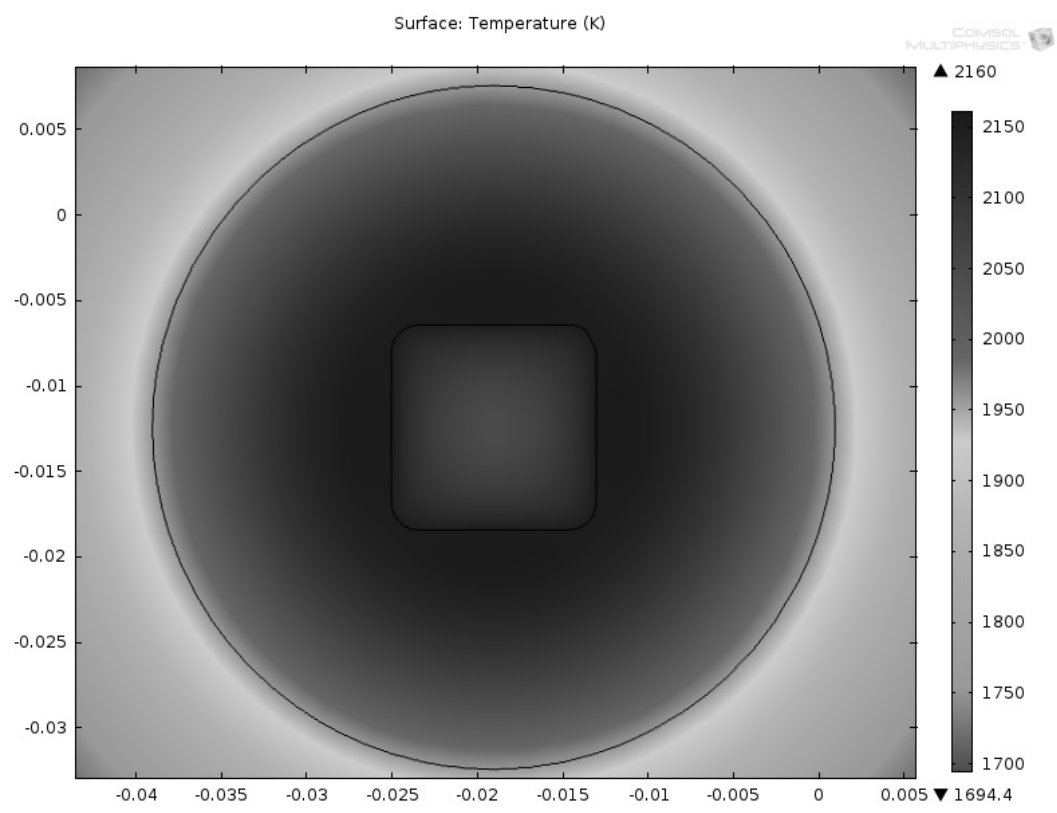

(b)

Fig. 11. Temperatures distribution during consolidation with shaping: (a) plates made of $\mathrm{WC}$, (b) plates made of $\mathrm{Al}_{2} \mathrm{O}_{3}$.

of technological equipment, covers, and semi-tools for nanocomposite semi-manufactured materials with preset parameters in full accordance with the requirements to engineering product samples.

Acknowledgements. The work was carried out within the framework of the Regulation of the Government of the Russian federation dated 9 April 2010 No. 220 "On measures taken to attract key scientists to Russian educational institutions of higher professional education, scientific establishments of state academies of science and state scientific centers of the Russian Federation" (Agreement No. 14.B25.31.0012 dated 26 June 2013).

\section{References}

[1] H. Yang, Multi-field simulation of the spark plasma sintering process, A Thesis in Engineering Mechanics by Hui Yang. The Pennsylvania State University, 2010, p. 154 
[2] K. Vanmeensel, A. Laptev, J. Hennicke, J. Vleugels, O. Van der Biest, Modelling of the temperature distribution during field assisted sintering, Acta Materialia 53 (2005) $4379-4388$

[3] J. Zhang, Numerical Simulation of Thermoelectric Phenomena in Field Activated Sintering, A Thesis for the degree of Ph.D., Drexel University, 2004, 169

[4] E. Aryan Khaleghi, Tailored Net-Shape Powder Composites by Spark Plasma Sintering, A dissertation submitted in partial satisfaction of the requirements for the degree Doctor of Philosophy in Engineering Science, University of California, San Diego, 2012, p. 231

[5] M. Ruskola, Numerical Modelling of Pulsed Electric Current Sintering Process, Thesis submitted for examination for the degree of Master of Science in Technology, Aalto University, Espoo, 2014, p. 95
[6] S.N. Grigor'ev, V.V. Kuzin, S.Y. Fedorov, T. Szalay, B. Farkas, Technological Aspects of the Electrical-Discharge Machining of Small-Diameter Holes in a High-Density Ceramic. Part 1, Refractories and Industrial Ceramics 55 (2014) 330-334

[7] V.V. Kuzin, S.N. Grigor'ev, V.N. Ermolin, Stress Inhomogeneity in a Ceramic Surface Layer Under Action of an External Load. Part 1, Effect of Complex Mechanical Loading, Refractories and Industrial Ceramics 54 (2014) 416-419

[8] V.V. Kuzin, S.N. Grigor'ev, V.N. Ermolin, Stress Inhomogeneity in a Ceramic Surface Layer under Action of an External Load. Part 2, Effect of Thermal Loading, Refractories and Industrial Ceramics 54 (2014) 4 97-501 